\title{
A PILOT-STUDY ON A TEXT MESSAGING-BASED MEDICATION COUNSELLING AND DRUG INFORMATION PROGRAM (PHARMATXTPINOY)
}

Maria Jayveelyn M Lalusis, ${ }^{1}$ Agnes May R Cayco, ${ }^{1}$ Imelda G Pena, ${ }^{2}$

April Czareen D Cruz'. 'Institute Of Medicine, FEU-NRMF, Quezon City, Philippines;

${ }^{2}$ University of the Philippines Manila College of Pharmacy, Manila, Philippines

10.1136/bmjopen-2015-forum2015abstracts.97

Background This descriptive study involves the development of a convenient and suitable program that can be adopted as a cognitive pharmaceutical service provided by community pharmacies in the Philippines.

Objectives This program, dubbed "PharmaTxtPinoy", aims to provide medication counselling services and reliable drug information to patients through text messaging.

Methods This study involved three phases, (1) the planning phase where surveys were conducted to identify the financialand time-concerns of possible program participants, specifically, patients with cardiovascular disorders in the Philippine General Hospital Out-patient Department and pharmacy students from the UP College of Pharmacy who served as drug informationproviders; (2) the implementation phase where the program developed by the researchers was executed, utilizing the FrontlineSMS version 1.6.16.3 software which is fully available online; and (3) the evaluation phase where the perceptions of the patients who inquired in the program were assessed through mobile phone interview.

Result Survey results showed that out of 212 patients, almost half $(43 \%)$ agreed to spend $5-10$ peso worth of mobile phone credits to inquire about their medications. Forty percent of the respondents were willing to wait for the responses to their queries within 5 minutes to 1 hour. For the survey on students, among the 71 respondents, majority (58\%) agreed to spend 30 minutes to one hour of their free time in participating in this kind of program, provided that there are academic or financial incentives. Further, the implementation phase was successfully conducted and all of the 14 patients who were evaluated, expressed positive feedbacks about the program.

Conclusion Employing a text messaging-based drug information and medication counselling program utilizing mobile phone innovations makes it easier for pharmacists to affirm their role as drug information provider. Patient counselling has also been made more accessible through this medium not only through its wider coverage but also through its capability to go beyond financial, geographical and educational barriers. The program generated positive feedback from the participants and direct recipients of drug information. And with this, the role and responsibility of the pharmacist is fulfilled.

Correction notice This abstract has been corrected since it was first published. An additional author has been included.

\section{REFERENCES}

1 Core-Lewis $\mathrm{H}$, Kershaw T. "Text messaging as a tool for behavior change in disease prevention and management", Epidemiologic Reviews, 2010;32, pp. 56-69, viewed 9 July 2011, <http://epirev.oxfordjournals.org/content/32/1/56.full.pdf>.

2 Langer A, Khumar B, Mittal A, et al. "Mobile Medicine: Providing Drug Related Information through Natural Language Queries via SMS". IEEE International Advance Computing Conference (IACC 2009). Patiala, India, 6-7 March 2009, viewed 11 March 2012 


\section{Abstracts}

3 Scott DM, Friesner DL, Miller DR. "Pharmacy Students" Perceptions of Their Preparedness to Provide Pharmaceutical Care", American Journal of Pharmaceutical Education 2010; 2010;74:(1) Article 8. viewed 11 March 2012,

4 Tolentino H, Marcelo A, Marcelo P, Maramba I. "Linking Primary Care Information Systems and Public Health Vertical Programs in the Philippines: An Open-source

Experience", AMIA 2005 Symposium Proceedings, 2005 pp. 311-315, viewed 9 July 2011. 